\title{
Multipresence-Enabled Mobile Spatial Audio Interfaces
}

\author{
Owen Noel Newton Fernando ${ }^{1}$, Michael Cohen ${ }^{2}$, and Adrian David Cheok ${ }^{1}$ \\ ${ }^{1}$ Keio-NUS CUTE Center, National University of Singapore, Singapore 119613 \\ \{newtonfernando, adriancheok\}@mixedrealitylab.org \\ 2 Spatial Media Group, University of Aizu, Aizu-Wakamatsu, Japan 965-8580 \\ mcohen@u-aizu.ac.jp
}

\begin{abstract}
Mobile telephony offers an interesting platform for building multipresence-enabled applications that utilize the phone as a social or commercial assistant. The main objective of this research is to develop multipresence-enabled audio windowing systems for visualization, attention, and privacy awareness of narrowcasting (selection) functions in collaborative virtual environments (CVEs) for mobile devices such as 3rd- and 4th-generation mobile phones. Mobile audio windowing system enhances auditory information on mobile phones and encourages modernization of office- and mobile-based conferencing.
\end{abstract}

\section{Introduction}

Vision and audition are the two main human senses for obtaining information about the outside world, and rich interfaces need both modes (at least!). Visual windowing systems allow multiple and multiwindow applications to share display resources; audio windowing systems, in analogy to graphical windows, can bring order to a cacophony of multiple simultaneous sound sources. Audio windowing can be thought of as a frontend, or articulated user interface, to a system with a spatial sound backend. Audio windowing systems are especially appropriate on small screen devices where there limited space for information and when control changes are not needed.

Researchers are working on interactive spatial audio to support synchronous groupware like teleconferences, either desktop applications or mobile device applications. For instance, Herder and Yamazaki [2] describe a networked virtual environment with 3D audio to support a chatspace while Aoki et al. [3] present a mobile audio space intended for use by gelled social groups. To the best of our knowledge, there is no existing presence awareness systems that can handle multiply present sources and sinks. The narrowcasting attributes [1] presented here suggest an elegant solution for such multipresence environments.

The suite of inclusion and exclusion narrowcasting commands for sources and sinks are like analogs of burning and dodging (shading) in photographic processing. The duality between source and sink operations is tight, and the semantics are identical: an object is inclusively enabled by default unless, a) it explicitly excluded (with mute or deafen), or, b) peers (either self-designated or non 
self-designation of the selected object) are explicitly included (with select [solo] or attend) when the respective avatar is not. We have developed a mobile phone interface which allows users to multicast their voice to multiple receivers and control crowded soundscapes using these narrowcasting operations. Programmed with J2ME, our application runs on NTT DoCoMo iappli mobile phones.

\section{2 " $\imath$ Con": Mobile Device Multispace Interface}

Featuring selectable icons with one rotational and two translational degrees of freedom, the " $\imath$.Con" $2.5 \mathrm{D}$ dynamic map interface is used to control position, sensitivity, and audibility of avatars in a groupware session. Its isosceles triangle icons are representations of symbolic heads in an orthographic projection, including narrowcasting attributes.

We have developed symbolic representations of narrowcasting operations (as shown in Figure 1) for the mobile interface. In our mobile application, narrowcasting attributes graphical displays are triply encoded - by position (before the "mouth" for mute and select, straddling the "ears" for deafen and attend), symbol ('+' for assert '-' for inhibit), and color (green for assert red, yellow, and orange for inhibit). The attributes are not mutually exclusive, and the encoding dimensions are orthogonal. For instance, a sink might be first attended, perhaps as a member of some non-singleton subset of a spaces sinks, and then later deafened, so that both attributes are simultaneously applied.

The select and attend attributes are denoted by characteristic features. If any avatar has been selected, non-selected avatars are implicitly muted if their self-designation state is the same as the selected avatar. For example, if a selfdesignated avatar is selected, only other self-designated avatars are implicitly muted. In the same manner, if any attended avatars exist in a given space, nonattended avatars are implicitly deafened if they are in the same class (self or non-self) as an attended avatar. Orange color is used to represent these implicit effects, implicit mute represented by a '-' before the mouth and implicit deafen by '-'s straddling the ears.

A unique feature of our system is the ability of a human pilot to delegate multiple avatars simultaneously, increasing quantity of presence; such multipresence enables one to overcome some fundamental constraints of human condition. Our interface will allow each human user to have sources and sinks present in multiple conversations at once. Users will be able to receive multiple audio streams and transmit their voices to multiple recipients, controlled according to custom preferences. Multiple sources are useful, for instance, in directing one's remarks to specific groups, decreasing the granularity of audibility control. Multiple sinks are useful in situations in which a common environment implies social inhibitions to rearranging shared sources like musical voices or conferees, as well as individual sessions in which spatial arrangement of sources, like the configuration of a concert orchestra, has mnemonic value. 


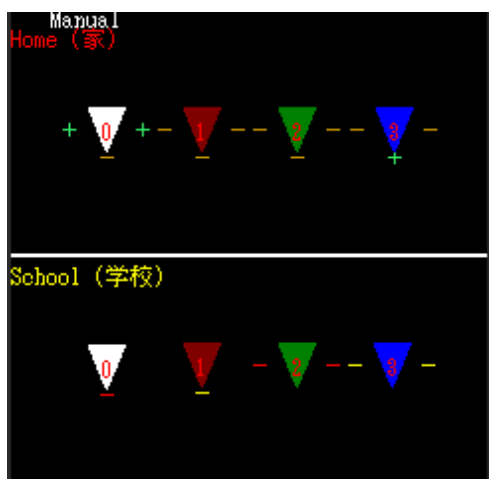

Fig. 1. Narrowcasting attributes graphical displays: In the Home space, avatar \#0 is attended, so its complement (comprising all the other avatars) is deafened; and avatar \#3 is selected, so its complement is implicitly muted. In the School space, avatars $\# 0 \& \# 2$ are respectively muted and deafened by self while avatars \#1 \& \#3 are respectively muted and deafened by others.

\section{Conclusion}

The basic goal of this research is to develop rich idioms for selective attention, presence, privacy, and narrowcasting for presence awareness applications for a nomadic device like a mobile phone. A multipresence feature using these idioms encourages users to install avatar representatives of themselves in several places and spaces at once. Spatial sound enhances the conferencing capabilities in collaborative virtual environments. Multipresence-enabled systems will enhance office communication and make it easier to coordinate work groups. The system also enhance human communication, allowing users to interact with friends, family, and colleagues "anytime anywhere." We expect that commercial development of this research will involve partnerships with network providers, who might license such technology to offer to their subscribers as an added value service.

\section{References}

1. Cohen, M.: Exclude and include for audio sources and sinks: Analogs of mute and solo are deafen and attend. Presence: Teleoperators and Virtual Environments 9(1), 84-96 (2000) ISSN 1054-7460

2. Yamazaki, Y., Herder, J.: Exploring spatial audio conferencing functionality in multiuser virtual environments. In: 3rd International Conference on Collaborative Virtual Environments, pp. 207-208. IEEE Press, San Francisco (2001) ISBN 1-58113303-0

3. Aoki, P.M., Romaine, M.H., Szymanski, J.D., Wilson, T.D., Woodruff, A.: The mad hatters cocktail party: a social mobile audio space supporting multiple simultaneous conversations. In: Proc. of the Conference on Human Factors in Computing Systems, Ft. Lauderdale, Florida, USA, pp. 425-432. IEEE Press/ACM Press (2003) 